\title{
Design-in-play: improving the variability of indoor pervasive games
}

\author{
Bin Guo • Ryota Fujimura • Daqing Zhang • \\ Michita Imai
}

(C) Springer Science+Business Media, LLC 2011

\begin{abstract}
Treasure is a pervasive game playing in the context of people's daily living environments. Unlike previous pervasive games that are based on the predefined contents and proprietary devices, Treasure exploits the "design-in-play" concept to enhance the variability of a game in mixed-reality environments. Dynamic and personalized role design and allocation by players is enabled by exploring local smart objects as game props. The variability of the game is also enhanced by several other aspects, such as user-oriented context-aware action setting and playing environment redeployment. The effectiveness of the "design-in-play" concept is validated through a user study, where 15 subjects were recruited to play and author the trial game.
\end{abstract}

Keywords Multimedia entertainment · Pervasive gaming · Context-aware computing - Smart homes $\cdot$ Smart objects $\cdot$ Human-computer interaction

B. Guo $(\bowtie)$

School of Computer Science, Northwestern Polytechnical University, Xi'an, China e-mail: guobin.keio@gmail.com

B. Guo $\cdot$ D. Zhang

Institut TELECOM SudParis, 9, rue Charles Fourier, 91011 Evry, France

B. Guo

e-mail: bin.guo@it-sudparis.eu

D. Zhang

e-mail: daqing.zhang@it-sudparis.eu

R. Fujimura $\cdot$ M. Imai

Department of Information \& Computer Science, Keio University, Tokyo, Japan

R. Fujimura

e-mail: fujimura@ayu.ics.keio.ac.jp

M. Imai

e-mail:michita@ayu.ics.keio.ac.jp 


\section{Introduction}

The development of wireless sensor networks (WSNs) has propelled various innovative applications in smart environments. Pervasive gaming, a new genre in the field of entertainment, is one of its productions. By blending of real and virtual elements and enabling users to physically interact with their surroundings during the play, people can become fully involved in pervasive games and attain better gaming experience [15].

A number of projects or prototypes on pervasive games have been built $[2-8,16]$ (see Section 2 for details). Existing systems have greatly contributed to pervasive gaming research by emphasizing different natures of ubiquitous interaction. However, as the contents and game settings in these games are all predesigned by game developers (we call it the "design-before-play" paradigm), the variability of them is quite low, which may lead negative user experience to players during repeated plays. Broadly speaking, a typical pervasive game consists of five key elements: the playing field, physical props (roles acted by physical objects, like a magic wand), roles acted by players, game rules, and multimedia contents. Benefiting from the pervasive nature of pervasive games, the playing field can be changed from different places (e.g., from one room to another $[3,6,16]$, from one street to another $[2,4,7])$. Nevertheless, because the physical props used in these games are proprietary (particular designed flags [5], cottage and hand icons [16], etc.), they should be deployed into the new environment when the playing field changes. In addition, as the props are proprietarily offered and limited on numbers, players are not allowed to add new prop-based roles. The unchangeable props reduce the variability of the games. The latter three elements, on the other hand, are logical or virtual contents pre-specified or provided by game developers, which are also unalterable to players.

The system presented in this paper, called Treasure, makes an attempt to address the issues mentioned above. Treasure is a treasure-hunting game playing in the context of people's daily life. Different from the previous "design-before-play" paradigm enabled games, it seeks to improve the pervasive game's variability by introducing the "design-inplay" concept, which allows people to generate variations of a meta-game before playing it. The following attempts were made to achieve a big variability:

- Dynamic role design and allocation by employing local everyday objects. According to Weiser's vision on ubiquitous computing [19], homes in the near future will be filled with sensor-augmented everyday objects, called smart objects. By analyzing the data from the attached sensors (e.g., location sensors [11], acceleration sensors [1], pressure sensors [18], RFID tags [20]), smart objects can provide various assistance to people's life, such as helping people quickly locate them $[11,20]$, or identifying indoor human behaviors $[1$, 18]. Unlike providing particularly-designed game props, we exploit the prospect of using smart objects in pervasive games. Leveraging local smart objects as game props provides the following merits: (1) The normal, plentiful nature of smart objects allows users to dynamically add new prop-based roles into the game and select interested objects to play the new roles (e.g., choosing a smart pen to play the 'magic wand' role); (2) it enhances the variability of the game by letting different objects to play the same role (replacing the smart pen by a smart phone to play the 'magic wand' role).

- Player configurable context-aware action setting. Players play the game by physically interacting with prop-based roles distributed in the house, such as moving a 'magic wand' or opening a drawer. For each detected human-prop interaction context, a player can specify a preferred multimedia action to respond (e.g., playing a narrative or displaying an adventure animation). 
- Reconfigurable game environment setting. The game environment consists of a playing field and physical props. As the whole smart house is used as the playing field, players can reconfigure the game environment by rearranging the object-based props in the house, for example, placing a prop to a particular place (e.g., hiding the 'magic wand' in a drawer), etc.

To the best of our knowledge, research on playing and player-authoring of smart-object based games has not been explored by previous studies. In the following sections, we firstly review related work on pervasive gaming. The Treasure game will then be described, followed by the technical support for the implementation of this game. The effectiveness of our system is evaluated through a user study, with users to perceive and test different support to enhance game variability.

\section{Related work}

Different approaches and varied forms have been explored in developing pervasive games. One approach is to augment traditional, real-world games with computing functionality. The enabled games include smart toys [8], storytelling games [3, 16], and augmented outdoor games [5]. Another approach, on the contrary, attempted to map computer games onto realworld settings, as demonstrated by Touch Space [6] and Human Pacman [7]. There are also a few location-aware games $[2,4]$, where a building, a street, or even a city becomes a game board and the players become playing pieces. Treasure follows the first approach, which aims at augmenting traditional treasure hunt game with computing functionalities.

There are several types of treasure hunt games which can have one or more players who try to find hidden articles or places by using a series of clues. Gold Miner (http://www. goldminergame.net/) is a video-based treasure hunt game designed for children. The locationaware game presented in Chalmers et al. [4] allows players to collect virtual coins that are hidden in the game area based on GPS information. Compared with these systems, the Treasure game provides better user experience by allowing them to hunt physical 'treasures'.

There have been several studies that examine pervasive gaming in normal, daily settings. SupaFly [13] is a mobile-phone based virtual community game, where players can create characters and interact through them by sending SMS commands. The player's goal is to reach the highest level of status in the community by socially interacting with others (e.g., meeting nearby players, making new friends). NEAT [21] is a pervasive game that seeks to encourage physical activity in the daily routing of players. Data from player-equipped sensors is logged to the cell phone and control the animation of an avatar that represents the player in a virtual race game with other players over the cellular network. These systems demonstrate well how pervasive games affect our daily livings. Treasure is also a game playing in daily settings. Different from the prior two systems, we particularly concern how plentiful everyday resources and user participation can extend the variability of game experiences in smart homes.

User content creation is not new to video games, which has been used in commercial games like Tank 1990 (developed by Nintendo), Counterstrike (a first-person shooter game), and academic game studies like Storytelling Alice [14]. Users are either allowed to create game maps or author stories in them. Treasure extends the user creation concept used in these video games by enabling users to handle a number of gaming elements in mixedreality environments, such as everyday-object based role design and real-world game environment setting. Due to the nascent of the field, there are quite few studies about user 
creation in pervasive games. StoryRoom [16] is a storytelling game that allows children to create simple If-Then rules that facilitates story telling. The game is played in a particular designed storytelling corner and limited proprietary props are provided. The context-aware rules are created through a physical programming method, which can build connections between a prop-touch event and an actuator-based action. The EU-IP iPerg project [12] explores a text-messaging method that allows players to remotely control the activities of their "avatars" (e.g., changing locations/destinations, communicating with other players) on a physical game board in the "Day of the Figurines" game. There are also on-the-spot operators (co-producers) that can modify the game state, give response to messages from players. Comparing with StoryRoom and iPerg, Treasure opens up a wider space to increase game variability. First, it extends the game field from particular-designed game board to the whole end users' living environments (i.e., smart houses). Second, considering that different houses have different layout and physical/virtual resources (smart objects, multimedia contents, etc.), exploring smart objects as props and allowing multimediaaction settings enhances the diversity of enabled games. Third, the scope of participants on game authoring is much wider in our system. StoryRoom is designed for children for storytelling. iPerg, however, explores fixed operators (staffs, game designers) while not normal players to co-produce the game. Finally, our system allows users to freely add new roles (by exploring various smart objects in smart houses). The two prior systems only allow children to alter the behaviors of existing roles.

\section{The treasure game}

The logic of this game is simple: At the beginning of the game, objects are hidden in different places of the house. Different objects play different roles in the game, and when the players find a hidden-object, the relevant multimedia action is presented to transmit information to the players. Players need to hunt the 'treasure' to win the game.

The whole gaming process consists of two stages: game authoring stage, and game play stage. "Design-in-play" - the concept that allows end users (not developers) to create or modify the game contents when playing it—is reflected in the two stages:

- In the game authoring stage, a group of end users, called game authors, are allowed to reconfigure the settings of the game according to their imagination and preferences, such as deciding which objects are to be hidden, where to hide, and define their actions in response to human interaction events;

- In the game play stage, another group of end users, called players, play the game configured by game authors, whose goal is to "hunt" the treasure designated in the game authoring stage.

\subsection{Game authoring}

In this stage, the game author configures the game setting through the following three authoring tasks.

(1) Role design and allocation. The game author selects a few objects to play different prop-based roles in the game. There are two types of prop-based roles: target roles and supporting roles. 
- Target roles. There are two target roles in the game, including the 'treasure-box' and the 'treasure-box-key'. As the name suggests, if the player finds the target roles, he wins the game. Though the two target roles are fixed, the game author can choose different objects to act the two roles. As the example shown in Fig. 1, a $C D$ Box and a Bicycle-Key are used to act as the 'treasure-box' and 'treasure-box-key', respectively. From this example we can see that the relationship between the two selected objects is just a weak, imagined relation, that's to say, they do not have to have the real "key-to-lock" relation as existing between the target roles.

- Supporting roles. Different from target roles, the supporting roles are designed by game authors, whose responsibility is to (1) assist players to find target objects (i.e., a 'guide' role), or (2) provide funny or risky gaming experiences to players. One or more supporting roles can be added. For example, for the prior case, a doll can be used to act as a 'guide' that cues the player about the hidden place of the 'treasurebox'; while for the latter case, a toolbox can be imagined to be the 'shelter of a monster' and when it is opened, a monster residing in it will come out to frighten the player. The two examples are illustrated in Fig. 1.

(2) Context-aware action setting. The game author specifies diverse multimedia actions in response to the different human-prop interaction events (e.g., when an object is found by a player) occurred in a game play. In Treasure, the actions can be defined in different forms, such as an audio clip, a video clip, an image, or a combination of them. Taking the two supporting roles shown in Fig. 1 for example, for the Player-Doll interaction event, an video-action can be added, which cues the player to search around the cup for a target object; for the Player-Toolbox interaction event, a combination of an image-action and audio-action is added, which displays an image about a monster on the wall, accompanying with a voice like "Don't touch me, I am sleeping now". As shown in Fig. 2(1), a rotatable presentation device, called Prot, is used for displaying the multimedia contents to proper places (see Section 4.1 for details).

(3) Game environment setting. Before starting a game, the game author resets the game environment through the following ways:

- They hide the selected props in the "hidden places" of the house. As shown in Fig. 2(2), objects can be hidden in different places, such as on a shelf, inside a box,

\begin{tabular}{|c|c|c|c|c|c|c|}
\hline & $\begin{array}{c}\text { Events } \\
\text { (Question) }\end{array}$ & $\begin{array}{l}\text { Objects } \\
\text { (Roles) }\end{array}$ & & etting & Audio Setting & Hidden Place \\
\hline \multirow{5}{*}{ 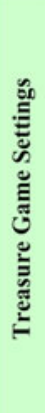 } & $\begin{array}{l}\text { Find an object } \\
\text { (Q1) }\end{array}$ & $\begin{array}{c}\text { A bicycle-key } \\
\text { (target role: treasure-box-key) }\end{array}$ & GIF & & $\begin{array}{l}\text { "You have found the } \\
\text { treasure-box-key." }\end{array}$ & Under a cup \\
\hline & $\begin{array}{l}\text { Find an object } \\
\text { (Q1) }\end{array}$ & $\begin{array}{c}\mathrm{A} \mathrm{CD} \text {-box } \\
\text { (target role: act as treasure box) }\end{array}$ & JPEG & & $\begin{array}{l}\text { "You have found the } \\
\text { treasure-box" }\end{array}$ & On a shelf \\
\hline & $\begin{array}{l}\text { Find an object } \\
\text { (Q1) }\end{array}$ & $\begin{array}{c}\text { A doll } \\
\text { (supporting role: act as a guide) }\end{array}$ & Flash & & - & In a drawer \\
\hline & $\begin{array}{c}\text { Find an object } \\
\text { (Q1) }\end{array}$ & $\begin{array}{c}\text { A toolbox } \\
\text { (supporting role: act as the } \\
\text { shelter of a monster) }\end{array}$ & GIF & & $\begin{array}{l}\text { "Do not touch me! I } \\
\text { am sleeping now" }\end{array}$ & Under a chair \\
\hline & $\begin{array}{l}\text { Win the game } \\
\text { (Q2) }\end{array}$ & $\begin{array}{l}\text { The bicycle-key } \\
\text { The CD-box }\end{array}$ & Flash & & $\begin{array}{l}\text { "You win this game. } \\
\text { Congratulations!" }\end{array}$ & - \\
\hline
\end{tabular}

Fig. 1 An authored treasure game 


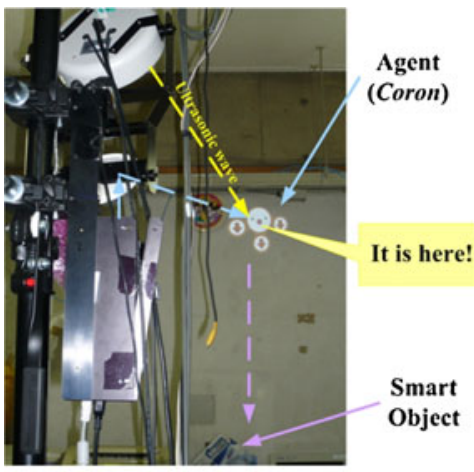

1. A guide agent is displayed on the wall by Prot device

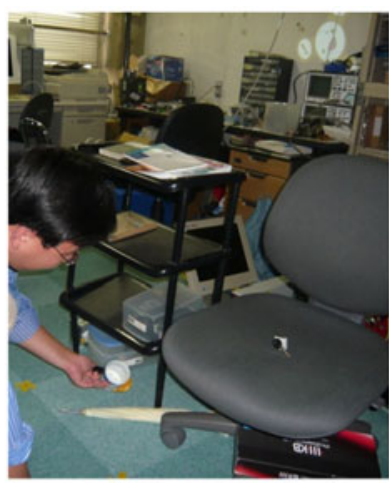

2. A player is hunting the treasure.

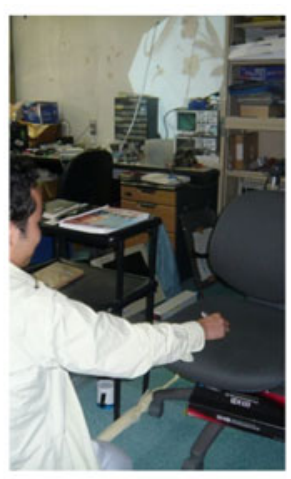

3. A player places an object he found on TEC.

Fig. 2 The co-location play mode

under a cup or a book. Since there could be 'guide' roles in the game, the hidden place of a relevant object in the real world should be consistent with its virtual presence in the game. For instance, in the example shown in Fig. 1, the BicycleKey should be placed under a cup according to the action setting of the dollguide' role.

- They can change the layout of the objects in the house, such as moving a shelf from one place to another, moving a box to a shelf, etc. In Fig. 2(2), a shelf which was placed in the corner is moved to the middle of the playing field.

\subsection{Game play}

According to whether the two types of end users play together in the same place, two play modes were developed: co-location mode and networking mode.

(1) Co-location play mode. In this mode, the game author and players are in the same place. For example, the mother from a family can author the game and ask her children to play it. The game starts with an introduction about the rules of the game. During the playtime, the player moves freely around the house to touch different objects. Once an object is found, it should be placed on a magic "Treasure-Evaluation Center" (TEC for short) to determine the identity of this object. In the example game play shown in Fig. 2(3), the surface of a chair is defined as the TEC. To eliminate conflicts, the TEC only identifies one object at a time (i.e., players cannot put two more objects on the chair in the meantime). Once the identity of an object is determined, the relevant virtual action will be triggered in response to the object-found event. For example, when a supporting object like a 'guide' is found, an agent will be displayed on the wall in front of the player to guide the player to a target object (see Fig. 2(1)). The game ends when the player finds both the two target objects. It also ends if the players cannot attain the goal within the given time (e.g., $5 \mathrm{~min}$ ).

(2) Networking play mode. This is an inter-home play mode, where the game author and player are at different places. For example, Lily sets a game in her house (i.e., Lily's house is the playing field) and another player, called Tom, plays the game online from 
a remote house. Different from the co-location mode, during the playtime, Lily also participates in the game and provides assistance to Tom, as described later.

When the game starts, Lily stays in her house. Since Tom is in a remote player and he cannot touch the props directly, an alternative interaction mode is provided. As shown in Fig. 3(1), Tom appears in the playing field as a virtual avatar on the wall (supported by the Prot device deployed in Lily's house). By authorizing Tom to remotely control the rotating angle of the Prot device, he can move the avatar around the playing field (see Fig. 3(2)) and asks Lily to pick up an object by gesture and voice based communications (see Fig. 3(3)). When Lily picks up the object Tom points to, relevant multimedia content (defined by Lily) will be displayed both on the wall of Lily's house (see Fig. 3(4)) and on the control window of Tom side (see Fig. 3(5)). Obtaining the presented information, Tom continues performing other actions to reach the goal. The game ends when Tom finds the two target objects or the playtime is over.

\section{System design and implementation}

As demonstrated in the last section, the design of Treasure follows the "design-in-play" principle, which allows end users to alter the contents of the original game created by professional developers. We call the original game the "meta-game", which consists of a set of basic rules for detecting the contexts/events occur in human-prop interaction (e.g., the player picks up an object). As shown in Fig. 4, to support end user creation over the meta-game, an authoring kit is provided. The playing of a user-authored game is supported by a smart game environment. We will describe the implementation of these different components in the following subsections.

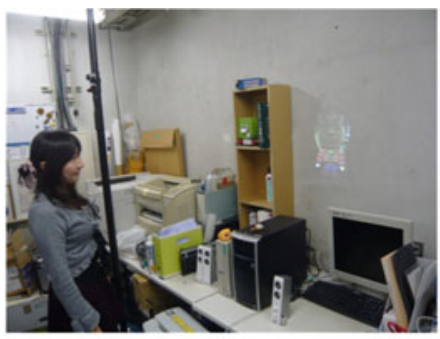

1. When the game starts, the avatar of the remote player appears on the wall.

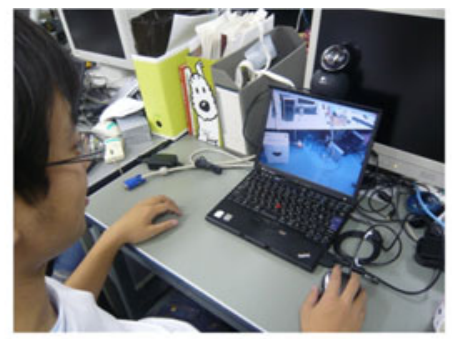

2. The remote player looks around the house for target objects.

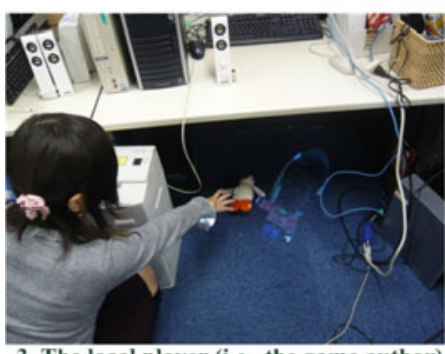

3. The local player (i.e., the game author) picks up the object pointed by the remote player

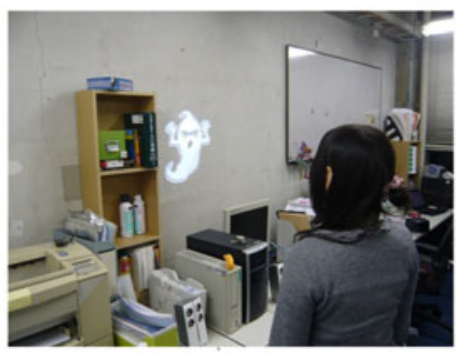

4. The multimedia action displayed on the wall of the playing field.

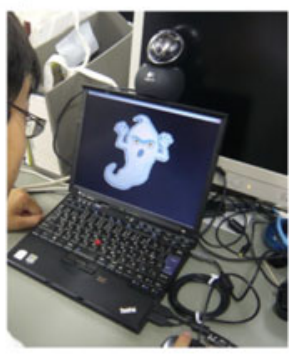

5. The remote player also views the action.

Fig. 3 The networking play mode 


\subsection{An experimental game environment}

To develop Treasure, we established an experimental smart home environment in our workplace using a combination of sensing and presentation techniques.

Indoor sensing techniques Various sensors are used to equip everyday objects, including ultrasonic 3D tags (U3D for short, see Fig. 5(a)), and MOTE sensors (a product of Crossbow, which is a combination of an acceleration sensor, light sensor, and sound sensor).

- The U3D location system, including U3D tags attached to objects and a number of ultrasonic receivers deployed on the ceiling (see Fig. 5(b)), leverages ultrasonic technique to locate indoor objects (please refer to our prior work [9] for details). By computing the difference of 3D-coordinates between the two or more updates from a U3D tag, we can infer the movement state of an object (e.g., it is picked up or moved by a player). In addition, because of the "limited coverage" problem $[9,11]$, an ultrasonicbased tag does not update data when it is not exposed to its readers (e.g., placed in a drawer or under a table), which can be indirectly used to detect the hidden/found status of an object.

- MOTE sensors are used to monitor the orientation of an object (e.g., tilted) as well as the change of its surrounding environment (e.g., a player is talking near it).

Numerous prototypical smart objects are enabled, and some examples are illustrated in Fig. 5(c) and (d).

Presentation techniques The most technically advanced pervasive games use augmented reality (AR) techniques as a basis to present virtual information to players [15]. In Treasure, we also explored an AR device, called Prot (see Fig. 2(1)), which is a combination of $a$ projector, an ultrasonic speaker, a webcam, and a 3-DOFs rotating base. It works in different ways under the two play modes mentioned in Section 3.2.

- Co-location mode. All virtual contents are maintained locally in this mode. Based on the location data from the U3D location system and with the support of the rotating base, the projector displays information in the form of animations or images on the wall that the player is currently facing. The ultrasonic speaker generates voices accordingly: the ultrasonic wave transmitted by the speaker can go straight through the air, and be converted to audible sound when reflected by real-world objects (like walls and floor).

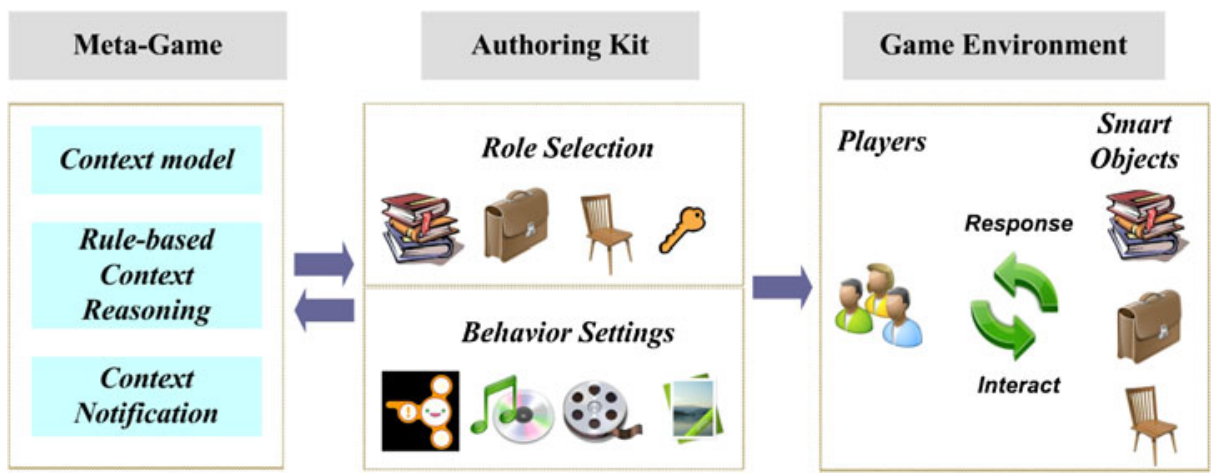

Fig. 4 Composition of the treasure game 


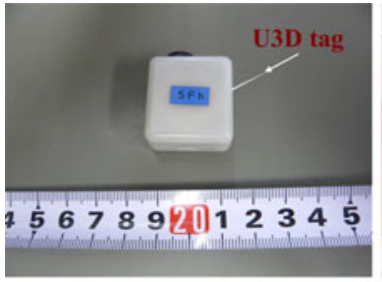

a Ultrasonic location sensor (U3D)

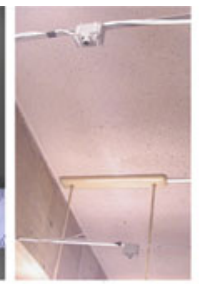

b Ultrasonic receivers on the ceiling

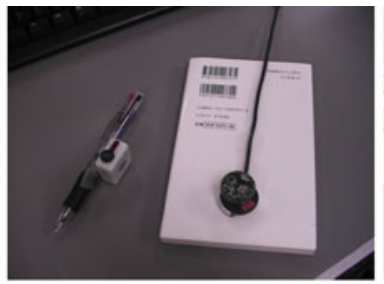

c Smart pen, smart book (MOTE)

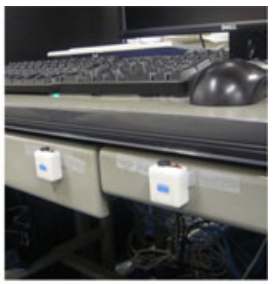

d Smart

drawers

Fig. 5 Sensors and smart objects

It makes players feel that the voices are coming from these objects (please refer to [17] for technical details).

- Networking mode. The virtual contents are transmitted among smart homes. As shown in Fig. 6, the webcam installed on the head of the Prot device is responsible for capturing the video information of the game environment and transmitting it to the remote player; while the projector and the ultrasonic speaker on Prot are used to present the live video/audio information of the remote player (captured by another webcam and a speaker installed on the remote user's side) in the form of his avatar in the playing field. The avatar has two motions, standing and pointing, which can be controlled through the remote user's mouse-click action. The movement of the avatar, on the other hand, is controlled through the mouse-dragging action.

This Prot device enables players to view and interact with "real-world" agents and avatars without using any head-mounted devices, which prohibits the issues relevant to them, such as occlusion [15] and uncomfortable feelings from players. Example are shown in Figs. 2(1) and 3(1), where the player feels that he is having a face-to-face conversation with the "on-wall" agent or a "remote" friend.

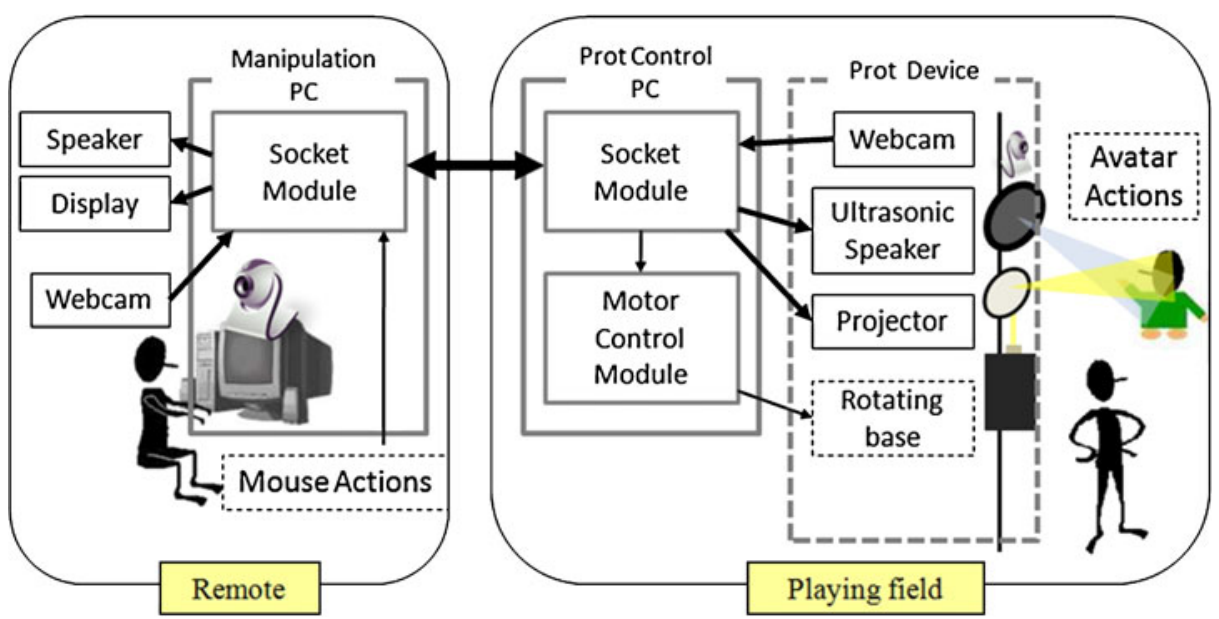

Fig. 6 Technical support for the player-avatar 


\subsection{Meta-game design}

The meta-game contains several basic modules of the game, which is supported by the Sixth-Sense infrastructure. Sixth-Sense is a context infrastructure which supports rapid prototyping of human-object interaction systems (refer to our prior work for details [10]). It provides the following support for building the Treasure system:

- The ontology-based context model. The Sixth-Sense infrastructure adopted the ontologybased method for context modeling. The ontology, called SS-ONT, is built using the Semantic Web language-OWL. The SS-ONT home domain ontology defines a set of concepts and relationships that exist in a typical smart home environment, such as humans, smart objects, and the relationship between humans and objects. To include new concepts used in the Treasure game, such as the hidden/found status of smart objects and the win/ loss status of the game, we extend the domain ontology by adding these new terms.

- Rule-based context reasoning. To support inferring high-level contexts (e.g., humanobject interaction events) from raw sensor data, a rule-based method is offered. Jess (http://www.jessrules.com/), a forward-chaining inference engine is used to execute inference rules. In Treasure, two particular contexts need to be detected: (1) the situation that a hidden object is found, and (2) the condition that a player wins the game should be defined. We explored the "limited coverage" weakness (see Section 4.1) to detect the hidden/found status of an object. The relevant rules are formulated in Eqs. 1 and 2 by using the Jess rule language.

$$
\begin{array}{r}
\text { Object }(? x) \wedge U 3 D(? y) \wedge \text { hasSensor }(? x, ? y) \wedge \\
\text { status }(? y, \text { notUpdated }) \rightarrow \operatorname{status}(? x, \text { Hidden }) \\
\text { Object }(? x) \wedge U 3 D(? y) \wedge \text { hasSensor }(? x, ? y) \wedge \\
\text { status }(? y, \text { Updated }) \wedge \operatorname{status}(? x, \text { Hidden }) \rightarrow \operatorname{status}(? x, \text { Found })
\end{array}
$$

The outcome of this game, i.e., the rule to determine if a player wins, is expressed by Eq. 3 .

Game $($ Treasure $) \wedge \operatorname{Object}(? x) \wedge \operatorname{Object}(? y) \wedge \operatorname{status}(? x$, Found $) \wedge \operatorname{status}(? y$, Found $)$

$\rightarrow$ GameStatus(Treasure, Win)

- Context notification. The Treasure game can subscribe some contexts (e.g., an object is found) from the context infrastructure. Once a particular event is detected, Sixth-Sense will notify the running application about it. Afterwards, the application can trigger the related context-aware action in response to the current situation.

\subsection{Game authoring kit}

As described in Section 3.1, the meta-game is authored through three tasks: role selection, context-aware action setting, and game environment reconfiguration. Among them, the latter one is performed in physical environment, whereas the prior two ones are achieved through a Web-based authoring kit. As shown in Fig. 7(a), the game authoring kit consists of two form-filling questions, each of which corresponds to a particular game event 
detected in Section 4.2: $(Q 1)$ the event that a hidden object is found, and $\left(Q^{2}\right)$ the status that the players win the game. The two tasks are weaved into the two questions.

- Role selection: In Q1, game authors can choose which objects are to be hided (all available smart objects in the house are listed in a selection box, as shown in Fig. 7(a)). Multiple roles can be added by repeatedly answer this question. In $Q 2$, the game author can specify two of the objects he chose in $Q 1$ to act the two target roles, for example, the Bicycle-Key and the $C D$-Box shown in Fig. 1. This, at the background level, will reshape the rule shown in Eqs. 3 to 4 (by replacing the variables ?x,?y to the names of the two selected objects).

$$
\begin{aligned}
& \text { Game }(\text { Treasure }) \wedge \text { Object }(\text { BicycleKey }) \wedge \text { Object }(\text { CDBox }) \wedge \\
& \text { status }(\text { BicycleKey, Found }) \wedge \text { status }(\text { CDBox, Found }) \\
& \rightarrow \text { GameStatus }(\text { Treasure, Win })
\end{aligned}
$$

- Context-aware action setting: In $Q 1$, the game author can specify which action is to be done when a hidden object is found. Three kinds of multimedia repository are provided: a video repository (Flash movie clips, video clips, etc., as shown in Fig. 7(b)), an image repository (JPEG files, Gif files, etc., as shown in Fig. 7(c)) and an audio repository (mp3, wav files). To ensure the openness of the game and the richness of available resources, users are allowed to submit and use the digital contents they generated as well (e.g., photos they took, videos they shot, Flash files they created, audios they recorded). An action setting example is given in Fig. 7. According to this setting, when the 'treasure-box' (acted by a $C D-B o x$ ) is found, a picture that describes a heap of coins will be presented, and the voice informs the player that "Congratulations, you have found a target object".

\section{Evaluation}

We conducted a series of experiments to evaluate Treasure. The main purpose is to explore the prospect of everyday-object based game play and analysis the effectiveness of the "design-in-play" concept to the variability of pervasive gaming. The playing field was the
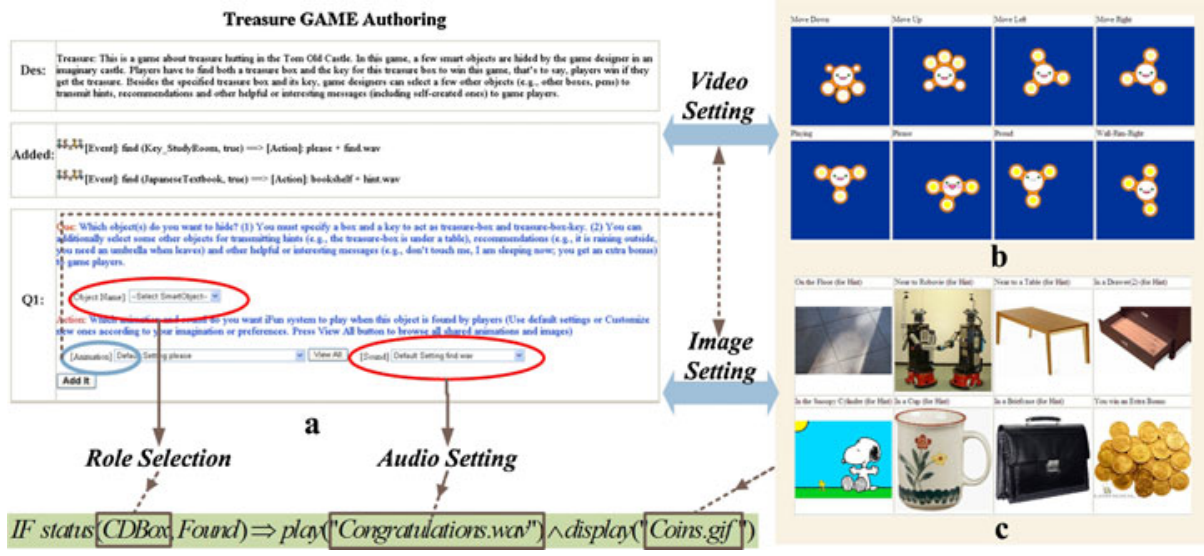

Fig. 7 The game authoring kit (a), animation-action repository (b), and image repository (c) 
experimental game environment (see Section 4.1) established in Keio University. During a total of two-month's testing period, we recruited by email 15 participates from Keio to experience our system. The subjects varied widely in age (ranging from 21 to 34), gender (3 females), and discipline (13 non-computer students).

Our study had four phases. In the introduction phase, we introduced the participants to the smart-object based gaming in future homes and briefed them on how to play the Treasure game. The success of a game depends on how much the players enjoy playing the game and how strong they become emotionally attached to it. This aspect was evaluated in the user experience phase. In this phase, participants played the game for approximately $10 \mathrm{~min}$ and answered the questions aimed to measure their experiences playing the game. The game authoring phase explored users' interests on game authoring. Participants were asked to author a variation of Treasure according to their imagination and set it in the playing field. This phase lasted for around $20 \mathrm{~min}$ for each subject. In the final discussion phase, participants expressed their thoughts and suggestions on how to improve the game. The whole test session lasted for approximately $40 \sim 55 \mathrm{~min}$ for each subject.

\subsection{User experience}

In this testing phase, subjects were asked to experience the pre-authored Treasure game. They participated in the game through the co-location play mode, which allows them to be fully involved in the game (by physically interacting with the props and getting real-time response from the ambient environment).

Game settings The game follows the settings shown in Fig. 1. To make it not too difficult for players to find objects, we restricted the playing field to a $3 \times 2 \mathrm{~m}$ area, as shown in Fig. 2 (left). Besides the four selected "hidden" objects, there were several other objects within the playing field, such as a chair, a desk with drawers, a 3-tire shelf, a cup and several books, which were good "hidden places" for the "hidden" objects. The subjects were asked to place the object he found on the surface of the chair (i.e., the TEC mentioned in Section 3.2) to ensure that the ultrasonic signal it emitted could be detected by the location system.

Findings On the whole, the game's pace was fast in most trial runs. The player moved around the playing field and touched different objects deployed. The result for the number of people under different object-found sequences is listed in Table 1. It is not surprising that the relative big, target-like objects, including the Toolbox and the $C D$-Box, were more easily to be touched by the players. The two objects became the first-found object by many subjects (about 87\%). Two subjects (13\%) were interested in the doll and they firstly touched this object. The Bicycle-Key (placed under a cup) was much more difficult to be found, and it became the last-found object of most subjects (87\%). For the effect of the 'guide' role (i.e., the doll), it appeared in the whole tests 11 times (73\%), and among which, 9 subjects $(82 \%)$ found the relevant target object according to the hint transmitted. Two subjects omitted the hint from the 'guide' and it took them more time to find the key.

There were also some failure situations caused by hardware problems. For example, one tester did not place the room-key-equipped U3D tag upright on the table, which makes it still unable to be detected by our location system. Another source of failure is caused by the ultrasonic-signal interference between the U3D location system and the ultrasonic speaker, which sometimes results in error readings to the U3D location system. In this case, a hidden object may be wrongly asserted to have been found by a player. Failures like this happened twice during all the experiments. 
User ratings After an experience of the game, subjects were asked to rate Treasure on its attractiveness, prospect of application, and interactivity on a scale of 1 to 5 . The result shown in Fig. 8(a) and (b) indicates that about $80 \%$ participants (score $>4$ ) were excited about this game and most of them (93\%) were willing to play such games at home. The results reveal that it is promising to develop smart-object based games is future homes. Another of our aim was to evaluate the interactivity factors of this game. Figure 8(c) illustrates how testers judged the mixed-reality aspects of the game they experienced. It appears that most players felt that our system provided them with an adequate and wellbalanced mixture of the physical and virtual elements.

\subsection{Game authoring}

In this test phase, we wanted to determine whether subjects could create variations based upon the meta-game. This testing was performed by using Treasure's authoring kit (see Fig. 7). Subjects were asked to complete their authoring task following the introductions written on this page. After that, they were asked to arrange the game environment according to their authoring settings.

Findings During the testing process, we observed that most subjects could perform this task with little or no guidance, although the time taken to accomplish this task varied from subject to subject (ranging from 8 to $17 \mathrm{~min}$ ). Following are detailed findings.

- Role creation and action setting. The number of prop-based roles (i.e., the objects to be hidden) created in the subjects' settings ranged from 3 to 7. Most subjects successfully completed these tasks according to the given requirements. One good example of subject-authored games is shown in Table 2, within which a Room-Key and a YellowBox were selected to play the two target roles. The subject also added two interesting supporting roles: a 'guide' and a 'bonus giver'. The latter role could give the player an added bonus when it was found. There were still several subjects who did not perform the task according to the given requirements. One subject did not notice the requirement that "You must specify a box and a key to act as treasure-box and treasure-box-key" and he forgot to create a 'treasure-box-key' role (see Table 3). Two subjects did not specify

Table 1 Objects found sequence during game play

\begin{tabular}{llllll}
\hline Sequence ID & First & Second & Third & Fourth & Number of players \\
\hline S1 & CD-Box(T) & Doll & Bicycle-Key(T) & - & 4 \\
S2 & Toolbox & CD-Box $(\mathrm{T})$ & Doll & Bicycle-key $(\mathrm{T})$ & 3 \\
S3 & CD-Box(T) & Toolbox & Bicycle-Key(T) & - & 3 \\
S4 & Toolbox & Bicycle-Key(T) & CD-Box(T) & - & 1 \\
S5 & Toolbox & CD-Box(T) & Bicycle-Key(T) & - & 1 \\
S6 & Doll & Bicycle-Key(T) & Toolbox & CD-Box(T) & 1 \\
S7 & Doll & CD-Box(T) & Bicycle-Key(T) & - & 1 \\
S8 & CD-Box(T) & Toolbox & Doll & Bicycle-Key(T) & 1 \\
S9 & CD-Box(T) & Doll & Toolbox & Bicycle-Key(T) & 1 \\
\hline
\end{tabular}

(T) means the relevant object is a target object 


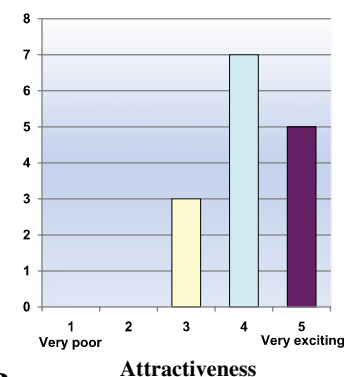

a (An overall impression of this game)

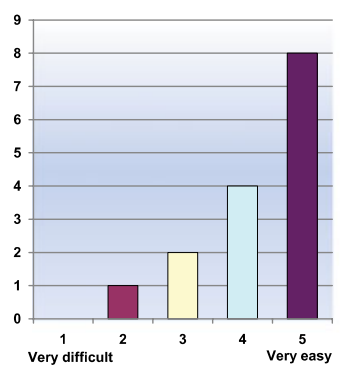

d

(Is it easy to author the game?)

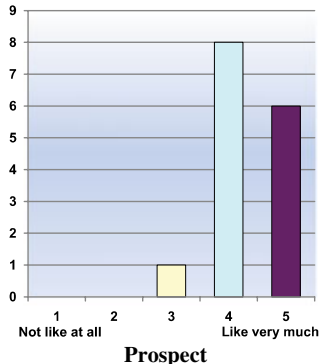

b

(Would you like to play such games at home?)

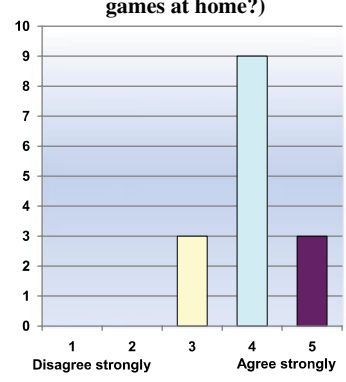

e Variability different gaming experiences)

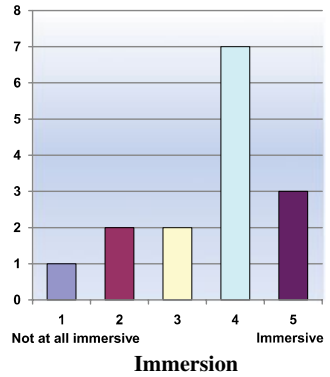

C (The game felt like a coherent whole despite different devices and interfaces)

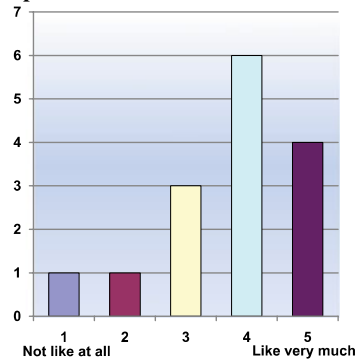

Willingness to design

(Would you like to participate in the game authoring process?)

Fig. 8 Experiment results

proper actions to the roles he added, such as the video setting for the 'treasure-box' role in Table 3.

- Game environment setting. Most subjects could follow their action settings when arranging the game environment. For example, the Yellow-Box is placed by the subject on a shelf according to the action setting in Table 2. There were some failure cases. One subject put the object in an exposed place (on the surface of a shelf). Three subjects did not set the game according to their action settings. For example, according to the setting shown in Table 3, the shelf should be placed on the right ride of the chair, but the subject did not do this in the real-world setting.

User ratings Subjects were asked to rank Treasure on its support for user creation. It can be seen from Fig. 8 (d) that $80 \%$ subjects thought it was easy to author the game by using the authoring kit. The effectiveness of the "design-in-play" principle, i.e., the variability of the game was also evaluated, where about $80 \%$ subjects (see Fig. 8(e)) agreed that the authoring kit could help them produce different gaming experiences. To further determine the attractiveness of the "design-in-play" paradigm to players, we asked testers whether they were willing to participate in the game authoring process. The result, shown in Fig. 8 (f), describes that two-thirds of them had interest in it.

\subsection{Discussion and feedback}

The participants all felt that smart-object based game play was highly innovative. Most of them believed that there would be a market for our system. For example, at least five 
Table 2 User authored game (1)

\begin{tabular}{|c|c|c|c|c|}
\hline Event & Object (Role) & Video setting & Audio setting & Hidden place \\
\hline Find an object & $\begin{array}{c}\text { Room-key } \\
\text { (Treasure-box-key) }\end{array}$ & FLASH & "Treasure key is found" & Under a book \\
\hline Find an object & $\begin{array}{l}\text { Japanese book } \\
\text { (Guide) }\end{array}$ & JPEG & "A hint for you" & In a drawer \\
\hline Find an object & $\begin{array}{c}\text { Yellow-box } \\
\text { (Treasure-box) }\end{array}$ & FLASH & "Treasure box is found" & On the shelf \\
\hline Find an object & $\begin{array}{c}\text { Snoopy-box } \\
\text { (Bonus giver) }\end{array}$ & GIF & "The Bonus" & Under the chair \\
\hline Game Wins & $\begin{array}{l}\text { Room-key, } \\
\text { Yellow-box }\end{array}$ & FLASH & $\begin{array}{l}\text { "Congratulations, you are a } \\
\text { rich guy now!" }\end{array}$ & - \\
\hline
\end{tabular}

subjects mentioned the commercially successful product Wii (http://wii.com), which also integrates physical activity into games. However, they also stated that our system would be more popular, because it enabled users to play games in a more random fashion and, more importantly, allowed user configuration of the games. The user study also suggests several directions to improve our system.

(1) User creation and social sharing. Positive feedback was gained over the "design-inplay" principle used in Treasure: "On my daughter's birthday, I can hide the birthday present and ask her to find it out", "such games can bring us much funny at family parties", etc. However, some suggestions on user creation were also presented. One subject asserted that "Compared with video games, the risk experience in the game is low". He suggested that "When a monster appears in the game play, the players have to physically or mentally struggle to beat it. For example, they may be asked to enter a 'ball-pass' session (passing a smart ball between players for a predefined number of times), or a 'puzzle-answering' session to win the monster'. This is a constructive suggestion, which on one hand reveals users' creativity, and on the other hand, implies that the "design-in-play" concept can be extended to offer users more power on content creation. Another subject asked us "can the game I created be stored and showed to my friends". There is no doubt that social networks have been prevalent among users. Though Treasure integrates a networking play mode to improve interhome social interaction between users, the users need more on this, such as a way to share the contents they created and the experience they have.

(2) Technical improvement. The hardware or software techniques used can be improved for Treasure. For example, for hardware, the projection system might not work well either in a very bright room or suffering from the occlusion problem. For software, there is some

Table 3 User authored game (2)

\begin{tabular}{|c|c|c|c|c|}
\hline Event & Object (Role) & Video setting & Audio setting & Hidden place \\
\hline Find an object & $\begin{array}{c}\text { Apple } \\
\text { (Bonus-giver) } \\
\end{array}$ & FLASH & "Bonus" & On the shelf \\
\hline Find an object & $\begin{array}{c}\text { Yogurt-box } \\
\text { (Treasure-box) }\end{array}$ & JPEG & "This is the Treasure-box" & On the shelf \\
\hline Find an object & $\begin{array}{l}\text { Teacup } \\
\text { (Guide) }\end{array}$ & FLASH & "Find here the treasure" & Under the chair \\
\hline Find an object & $\begin{array}{c}\text { Briefcase } \\
\text { (Fun-giver) }\end{array}$ & JPEG & "Not me, try again" & Under the chair \\
\hline Game Wins & Apple, Yogurt-box & FLASH & "You get the money" & - \\
\hline
\end{tabular}


latency (caused by positioning and context reasoning) on system response time to player activities (at a mean of $1.5 \mathrm{~s}$ ). One player suggested that, before real actions were performed, there should be a "Seeking..." like message displayed to players to inform them that the objects they found had been detected by our system. Evidently, device failure or technical shortcoming is a common problem to ubiquitous computing systems. We will take measures to cope with them in our future work, an object-relation based sensor failure detection mechanism had been proposed in our prior work [9].

(3) Design of more attractive user interface. Another reason for the players' dissatisfaction might be that the interface of the authoring kit was not visually appealing to game authors. Here are some of their answers on why they did not author the game according to the guide of the toolkit: "there are too many texts displayed on this page, which makes it easy to omit one or two sentences", "there should be more graphics". Treasure demonstrates our initial effort on validating the prospect of everyday-object based game creation. We agree that as an authoring kit for game, the interfaces should be intuitive and amusing to players. We intend to design a Web-based virtual house where users can visually author the game settings. Second Life (http://secondlife.com/) - a virtual world platform where users can virtually create virtual objects and customize their avatarprovides us a possible way to improve the interface.

\section{Conclusion}

Treasure represents our early efforts to increase the variability of pervasive games in mixedreality environments. The "design-in-play" principle, which allows end users to create or alter contents before each game play, is proposed to achieve this. To support dynamic and personalized role design, we explore the numerous smart everyday objects in smart homes as props. Treasure further allows players to configure the context-aware actions of the roles they created and reorganize the physical objects in the gaming environment. The evaluation results from an initial user study reveal that diverse gaming experiences can be generated and better user experience can be attained by allowing users to configure different elements of a meta-pervasive-game.

Based on the feedback from the subjects, we intend to make the authoring kit more userfriendly by introducing more graphical elements. In view of the fact that user content sharing has been a trend in the social networking era, we plan to create a general platform that enhances user cooperation and content sharing in the development of indoor pervasive games. Mechanisms will also be provided that allow users to create simple games and share their experiences with their peers.

\section{References}

1. Beigl M, Gellersen HW, Schmidt A (2001) Mediacups: experience with design and use of computeraugmented everyday objects. Comput Netw 35(4):401-409

2. Benford S et al (2006) Can you see me now? ACM Trans Comput-Hum Interact 13(1):100-133

3. Bobick AF et al (1999) The KidsRoom: a perceptually-based interactive and immersive story environment. Presence 8(4):369-393

4. Chalmers M, Bell M, Brown B, Hall M, Sherwood S, Tennent P (2005) Gaming on the edge: using seams in ubicomp games. In: Proc. ACM advances in computer entertainment, pp 306-309 
5. Cheok AD, Thang LN (2006) Capture the flag: mixed-reality social gaming with smart phones. IEEE Pervasive Comput 5(2):62-69

6. Cheok AD et al (2002) Touch-Space: mixed reality game space based on ubiquitous, tangible, and social computing. Pers Ubiquit Comput 6(6):430-442

7. Cheok AD et al (2004) Human Pacman: a mobile, wide-area entertainment system based on physical, social, and ubiquitous computing. Pers Ubiquit Comput 8(2):71-81

8. Fontijn W, Mendels P (2005) StoryToy the interactive storytelling toy. In: Proceedings of PerGames 2005, Munich

9. Guo B, Satake S, Imai M (2008) Home-Explorer: ontology-based physical artifact search and hidden object detection system. Mobile Inf Syst 4(2):81-103

10. Guo B, Satake S, Imai M (2008) Lowering the barriers to participation in the development of humanartifact interaction systems. Int J Semantic Comput 2(4):469-502

11. Harter A et al (2005) The anatomy of a context-aware application. In: Proc. of 5th ACM international conference on mobile computing and networking, pp 59-68

12. iPerG - integrated project of pervasive games, http://www.pervasive-gaming.org/index.php

13. Jegers K, Wiberg M (2006) Pervasive gaming in the everyday world. IEEE Pervasive Comput 5(1):78-85

14. Kelleher, C, Pausch, R, Kiesler, S (2007) Storytelling Alice motivates middle school girls to learn computer programming. In: Proc. CHI 2007, pp 1455-1464

15. Magerkurth C, Cheok AD, Mandryk RL, Nilsen T (2005) Pervasive games: bringing computer entertainment back to the real world. ACM Comput Entertainment 3(3):4-4

16. Montemayor J, Druin A, Chipman G, Farber A, Guha ML (2004) Tools for children to create physical interactive storyrooms. ACM Comput Entertainment 2(1):12-12

17. Nakadai K, Tsujino H (2005) Towards new human-humanoid communication: listening during speaking by using ultrasonic directional speaker. In: Proc. of robotics and automation, pp 1495-1500

18. Orr RJ, Abowd GD (2000) The Smart Floor: a mechanism for natural user identification and tracking. In: Proc. of CHI 2000, New York, pp 275-276

19. Weiser M (1999) The computer for the 21st century. ACM SIGMOBILE Mobile Comput Commun Rev 3(3):3-11

20. Yap KK, Srinivasan V, Motani M (2005) Max: human-centric search of the physical world. In: Proc. of SenSys'05, pp 166-179

21. Yuichi F, Konstantinos K, Colin P, Pradeep B, Ioannis P, James L (2008) NEAT-o-Games: blending physical activity and fun in the daily routine. ACM Comput Entertainment 6(2):1-22

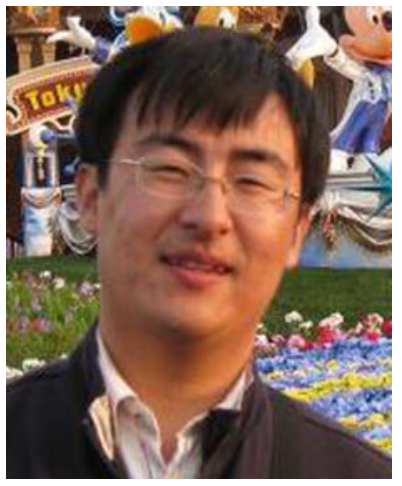

Dr. Bin Guo is currently an associate professor from Northwestern Polytechnical University, China, and a visiting researcher at Institute TELECOM SudParis, France. His research interest includes wireless sensor networks (WSNs), context-aware computing, pervasive gaming, and social intelligence. He received his $\mathrm{Ph}$. D. degree in computer science from Keio University, Tokyo, Japan, in 2009. He is a member of IEEE. 


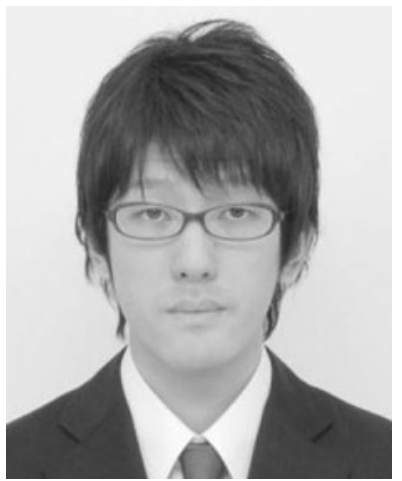

Mr. Ryota Fujimura is currently a master student at Anzai-Imai Lab., Keio University. His research interest includes augmented reality, group computing, and pervasive gaming.

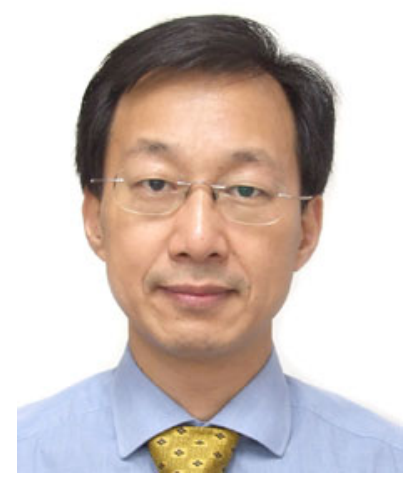

Dr. Daqing Zhang is a professor on Ambient Intelligence and Pervasive System Design at Institute TELECOM SudParis, France. He initiated and led the research in Smart Home, Healthcare/Elderly care and context-aware computing from 2000 to 2007 at the Institute for Infocomm Research (I2R), Singapore. Dr. Zhang was the Program Chair of the First International Conference of Smart Home and Health Telematics (ICOST2003) in Paris, France and the Sixth International Conf. on Ubiquitous Intelligence and Computing (UIC 2009) in Brisbane, Australia. He is the associate editor of ACM Transactions on Intelligent Systems and Technology, Journal of Ambient Intelligence and Humanized Computing (Springer), etc. He also served in the technical committee for conferences such as UbiComp, Pervasive, PerCom, etc. Dr. Zhang's research interests include pervasive elderly care, service-oriented computing, sensor based activity recognition, context-aware systems etc. He has published more than 100 papers in referred journals, conferences and books. Daqing Zhang obtained his Ph.D. from University of Rome "La Sapienza" and University of L'Aquila, Italy in 1996. 


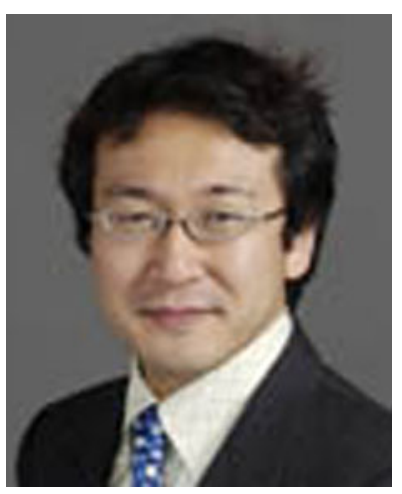

Dr. Michita Imai is Associate Professor of Faculty of science and technology at Keio University, a researcher at ATR Intelligent Robot Laboratories, and a visiting researcher at the University of Chicago. He received his Ph.D. degree in Computer Science from Keio Univ. in 2002. In 1994, he joined NTT Human Interface Laboratories. He joined the ATR Media Integration \& Communications Research Laboratories in 1997. His research interests include autonomous robots, human-robot interaction, speech dialogue systems, humanoid, and spontaneous behaviors. He is a member of Information and Communication Engineers Japan (IEICE-J), the Information Processing Society of Japan, the Japanese Cognitive Science Society, the Japanese Society for Artificial Intelligence, Human Interface Society, IEEE, and ACM. 